\section{Cureus}

Received 04/21/2019

Review began 06/04/2019

Review ended 06/07/2019

Published 07/04/2019

\section{(c) Copyright 2019}

Sourial et al. This is an open access article distributed under the terms of the Creative Commons Attribution License CC-BY 3.0., which permits unrestricted use, distribution, and reproduction in any medium, provided the original author and source are credited.

\title{
Thyroid Storm-induced Severe Dilated Cardiomyopathy and Ventricular Tachycardia
}

\author{
Kirolus Sourial $^{1}$, Saif M. Borgan ${ }^{1}$, Jorge E. Mosquera ${ }^{1}$, Loui Abdelghani ${ }^{1}$, Aamir Javaid ${ }^{2}$ \\ 1. Internal Medicine, University of Central Florida College of Medicine/Hospital Corporation of America \\ Graduate Medical Education Consortium, Orlando, USA 2. Cardiology, University of Central Florida \\ College of Medicine/Hospital Corporation of America Graduate Medical Education Consortium, Orlando, \\ USA
}

$\square$ Corresponding author: Kirolus Sourial, kirolus.sourial@ucf.edu

Disclosures can be found in Additional Information at the end of the article

\section{Abstract}

Thyroid storm is an extreme form of hyperthyroidism associated with a high mortality rate. Heart failure is considered the leading cause of mortality in patients with thyroid storm, though the underlying cardiac pathology is unclear. Approximately $6 \%$ of patients with thyroid storm have heart failure symptoms as the initial presenting complaint. Roughly, one-third of these patients develop dilated cardiomyopathy (DCM). In this report, we present a case of cardiogenic pulmonary edema and sustained ventricular tachycardia in a patient with hyperthyroidism presenting with thyroid storm.

Categories: Cardiology, Endocrinology/Diabetes/Metabolism, Internal Medicine

Keywords: thyroid storm, graves' disease, autoimmune myocarditis, non-ischemic cardiomyopathy.

\section{Introduction}

Cardiovascular manifestations of hyperthyroidism are related to decreased systemic vascular resistance and hyperdynamic status [1]. Hyperthyroidism is an uncommon cause of dilated cardiomyopathy (DCM) and heart failure. Burch-Wartofsky criteria are used in the diagnosis of thyroid storm [2]. If a patient develops cardiomyopathy without a known etiology, it is essential to assess the patient's thyroid function, as early treatment of hyperthyroidism can reverse the left ventricular dysfunction [1]. In this report, we present a rare case of thyrotoxic cardiomyopathy (TCMP) with severe left ventricular dysfunction. We also discuss possible pathopysiology of how hyperthyroidism can cause cardiomyopathy and explore currently available strategies in the treatment of patients with hyperthyroidism and cardiomyopathy.

\section{Case Presentation}

A 52-year-old woman with a past medical history of uncontrolled Graves' disease (GD) secondary to medication non-compliance presented to our emergency department with a oneweek history of worsening dyspnea and increasing anxiety symptoms. Upon evaluation, the patient was noted to be in moderate respiratory distress, tachycardic, afebrile, anxious and tremulous. The rest of the physical exam was remarkable only for bilateral fine rales up to mid lung zones. Electrocardiogram showed sinus tachycardia with a heart rate of 133 beats per minute (BPM) and nonspecific ST and T wave changes (Figure 1). 


\section{Cureus}

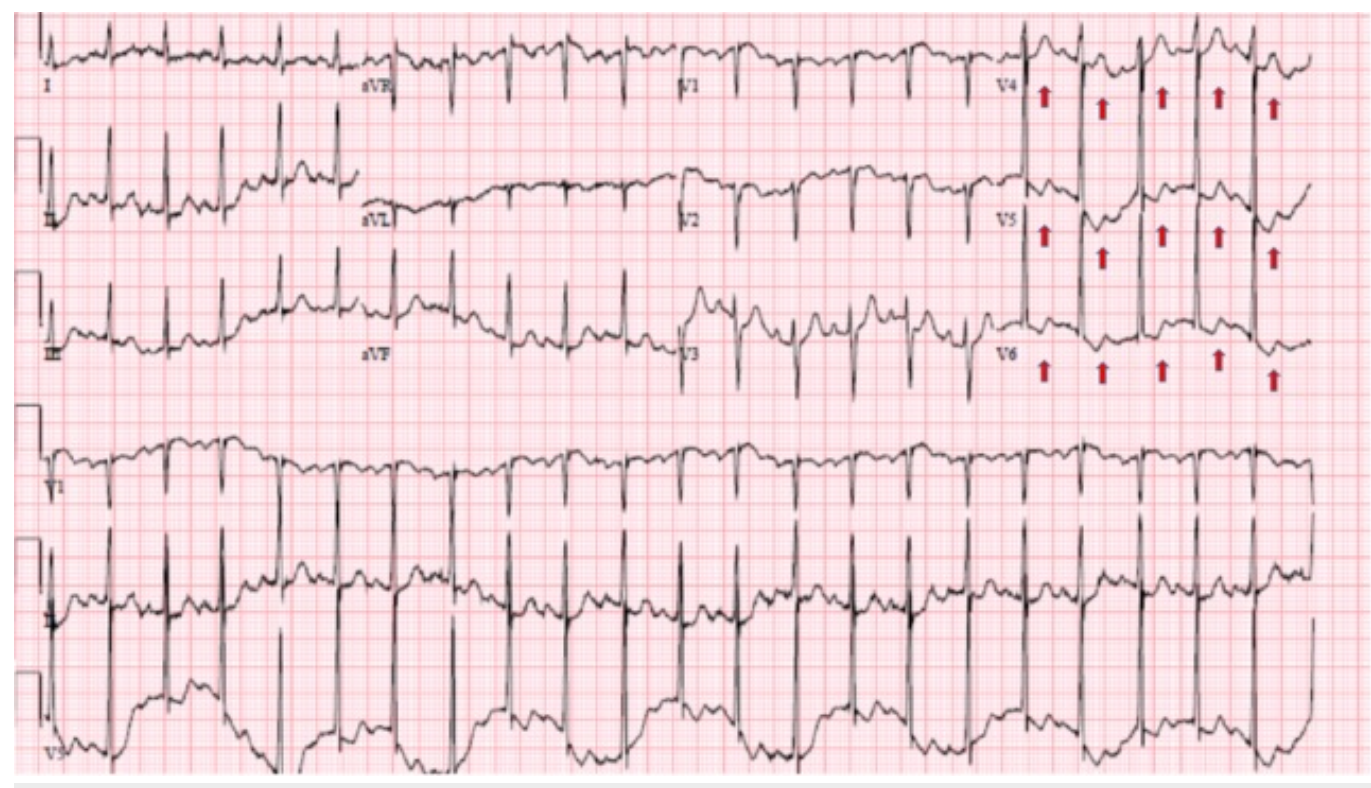

FIGURE 1: Electrocardiogram showing sinus tachycardia in addition to nonspecific ST and T wave abnormalities (red arrows) in leads V4- V6

Emergency measures included administration of Ativan and noninvasive respiratory support through bilevel positive airway pressure (BiPAP) resulted in minimal improvement. Blood test results showed extremely elevated free $\mathrm{T} 3$ and $\mathrm{T} 4$ levels $>20.00 \mathrm{pg} / \mathrm{mL}$ and $>8.00 \mathrm{ng} / \mathrm{dL}$ respectively, with suppressed thyroid-stimulating hormone (TSH) $<0.01 \mathrm{uIU} / \mathrm{mL}$ and positive thyroid antibody levels with thyroid peroxidase antibody $>1000.0 \mathrm{IU} / \mathrm{ml}$. Initial BurchWartofsky score was 35 . The patient developed flash pulmonary edema and sustained ventricular tachycardia. She required emergent cardioversion followed by endotracheal intubation and mechanical ventilation support. Post intubation chest X-ray confirmed the development of pulmonary edema (Figure 2). 


\section{Cureus}

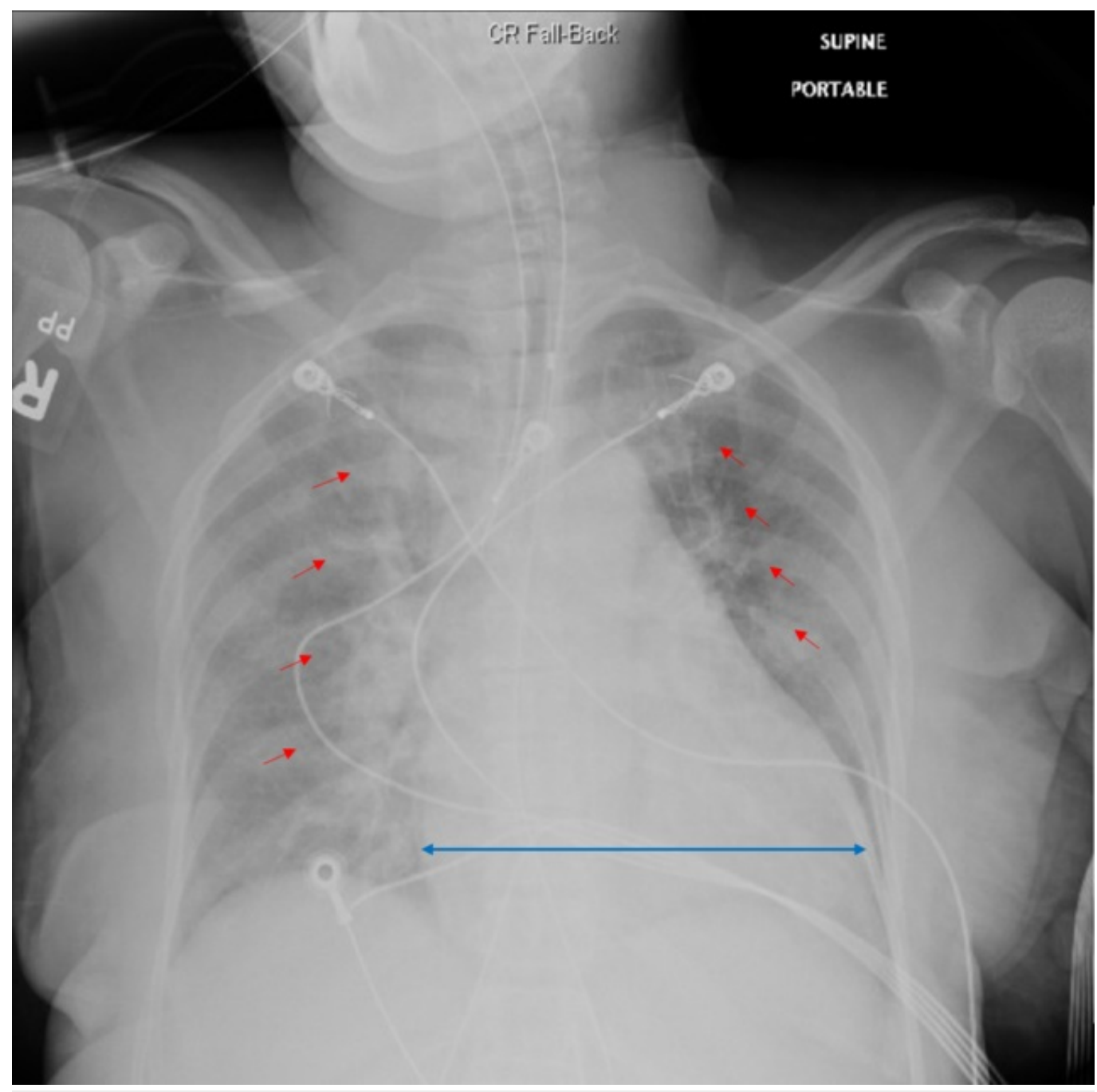

FIGURE 2: Post intubation chest X-ray showing increased bilateral airspace opacities which represent pulmonary edema (red arrows) and trace bilateral pleural effusions along with cardiomegaly (blue arrow)

Follow-up Burch-Wartofsky score was 55. Diuresis with intravenous Lasix was initiated along with propranolol, propylthiouracil, and corticosteroid therapy. Echocardiography was remarkable for severe DCM and severe global hypokinesis with an ejection fraction of $20 \%$ to $25 \%$. The patient's condition improved dramatically after 48 hours and she was successfully extubated. Her medical regimen was switched to oral carvedilol, tapering steroid dose, and methimazole. Prior to discharge, a pharmacological nuclear cardiac stress test ruled out the possibility of ischemic heart disease as an etiology of the cardiomyopathy.

The patient was provided with a life vest, and scheduled for follow up echocardiogram in three months.

\section{Discussion}

Thyroid storm in the setting of GD often presents after a precipitating factor such as surgery, infection, non-compliance with anti-thyroid medication, or radioactive iodine ablation, with prior illness being the most common trigger [2]. Our patient reported non-adherence to her 


\section{Cureus}

prescribed anti-thyroid medications due to financial hardship and inability to afford physician follow-up visits.

Burch-Wartofsky score is used to assess the probability of thyrotoxicosis based on clinical findings [2]. A score greater than 45 is highly suggestive of thyroid storm. Our patient had a score of 55 within hours of presentation. Refer to Table 1 for more information about BurchWartofsky score [3].

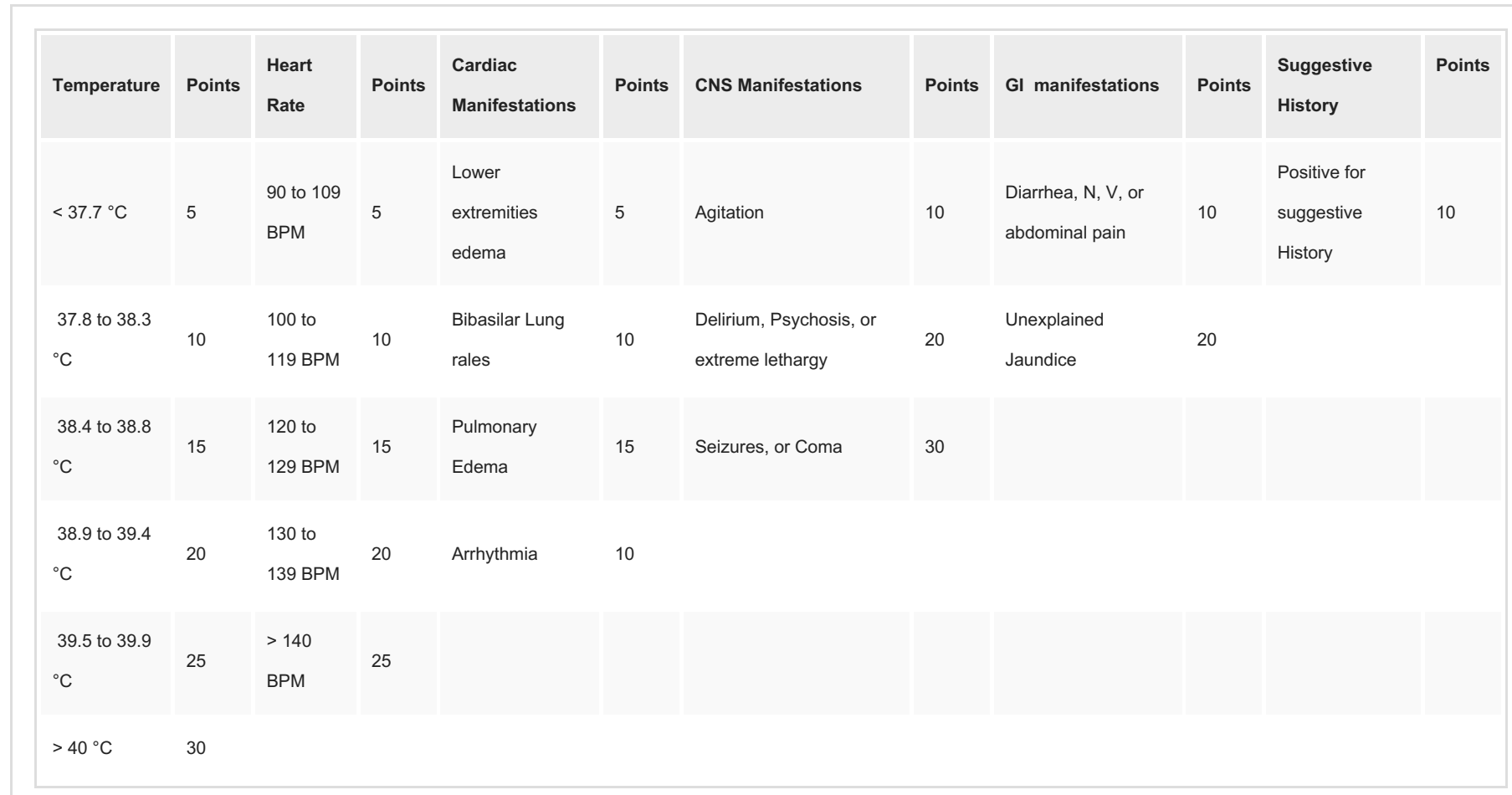

\section{TABLE 1: Burch-Wartofsky score}

A score of < 25: less likely to be thyroid storm, 25-44: suggestive of thyroid storm, and $>44$ : highly suggestive of thyroid storm.

C: celsius; BPM: beat per minute; CNS: central nervous system; GI: gastro-intestinal; N: nausea; V: vomiting.

Elevated thyroid hormone levels are thought to mimic adrenergic excess state by directly upregulating cardiac $\beta$ receptors and enhancing myocardial sensitivity to sympathetic and vagal innervation $[2,4-5]$. Thyroid hormone can also indirectly cause peripheral vasodilatation, decrease systemic vascular resistance, and increase in cardiac output by up to 300\% [2]. Hence, beta-blockers are a vital component of thyroid storm management. In patients with thyroid storm and congestive heart failure, non-selective beta-blockers should be used carefully to decrease the risk of heart failure exacerbation [6].

Irregular cardiac rhythms are thought to be the main cardiac manifestation in patients with thyrotoxicosis. However, less frequent manifestations such as severe cardiac dysfunction should also be considered. For example, hyperthyroidism-induced Takotsubo cardiomyopathy has been previously reported [7].

Unfavorable prognostic factors in TCMP include the age of onset, atrial fibrillation during hospitalization, and recurrences of thyrotoxicosis. Electrocardiographic changes seen in TCMP, 
such as T wave inversions, are commonly seen at the time of presentation and can be easily attributed to ischemia, which could delay the diagnosis and management and lead to unfavorable outcomes [8].

Despite the known association between thyroid storm and cardiomyopathy, the underlying cardiac pathophysiology is still not completely understood [9]. GD is a systemic autoimmune disorder [10]. One study reported an autoimmune mechanism could be contributing to the myocardial dysfunction in TCMP [1]. Lymphocytic myocarditis has been described in an autopsy of a refractory case of congestive heart failure secondary to hyperthyroidism [9]. The previous data support the use of steroids in some cases of thyrotoxicosis. For example, one case report showed that a patient with thyroid storm-induced severe cardiomyopathy had a dramatic recovery after steroid pulse therapy. It is believed that the anti-inflammatory effect of steroids improves cardiomyopathy induced by thyroid storm [1]. However, there are no available guidelines regarding the optimal formulation, dose or duration of steroids.

Anti-thyroid drug (ATD) therapy (propylthiouracil and thiamazole) can also improve the recovery of the cardiomyopathy by suppressing the thyrotoxic state and correcting the primary hemodynamic disturbances [4]. In one study, seven thyrotoxic patients with congestive heart failure showed an increase of mean left ventricular ejection fraction (LVEF) from $28 \%$ to $55 \%$ after ATD therapy [11]. Early diagnosis of these rare and potentially reversible cases is important to improve the heart function and patient outcomes. The traditional management includes ATD to suppress thyroid hormone synthesis and release, and beta-blockers to protect myocardial tissues against the harmful effect of thyroid hormones. Steroids are recommended in life-threatening thyroid storm, but their use in managing non-storm TCMP is unclear.

\section{Conclusions}

Early diagnosis of these rare and potentially reversible TCMP cases is important to improve the heart function and patient outcomes. Steroids use in managing non-storm TCMP is unclear. Further studies are needed to establish steroid effectiveness in lower-risk patients.

\section{Additional Information \\ Disclosures}

Human subjects: Consent was obtained by all participants in this study. Conflicts of interest: In compliance with the ICMJE uniform disclosure form, all authors declare the following:

Payment/services info: All authors have declared that no financial support was received from any organization for the submitted work. Financial relationships: All authors have declared that they have no financial relationships at present or within the previous three years with any organizations that might have an interest in the submitted work. Other relationships: All authors have declared that there are no other relationships or activities that could appear to have influenced the submitted work.

\section{References}

1. Kiriyama H, Amiya E, Hatano M, et al.: Rapid improvement of thyroid storm-related hemodynamic collapse by aggressive anti-thyroid therapy including steroid pulse: A case report. Medicine. 2017, 96:7053. 10.1097/MD.0000000000007053

2. Underland LJ, Villeda GA, Pal A, Lam L: A case of thyroid storm associated with cardiomyopathy and poststreptococcal glomerulonephritis. Case Rep Pediatr. 2016, 2016:1-3. 10.1155/2016/7192359

3. Viswanath O, Menapace DC, Headley DB: Methamphetamine use with subsequent thyrotoxicosis/thyroid storm, agranulocytosis, and modified total thyroidectomy: a case report. Clin Med Insights Ear Nose Throat. 2017, 17: 10.1177/1179550617741293 


\section{Cureus}

4. Eliades M, El-Maouche D, Choudhary C, Zinsmeister B, Burman KD: Takotsubo cardiomyopathy associated with thyrotoxicosis: a case report and review of the literature. Thyroid. 2014, 24:383-9. 10.1089/thy.2012.0384

5. Wu WT, Hsu PC, Huang HL, Chen YC, Chien SC: A case of takotsubo cardiomyopathy precipitated by thyroid storm and diabetic ketoacidosis with poor prognosis. Acta Cardiol Sin. 2014, 30:574-577. 10.6515/ACS20131218A

6. Ngo AS, Lung Tan DC: Thyrotoxic heart disease. Resuscitation. 2006, 70:287-90. 10.1016/j.resuscitation.2006.01.009

7. Radhakrishnan A, Granato JE: An association between Takotsubo cardiomyopathy and thyroid storm. Postgrad Med. 2009, 121:126-30. 10.3810/pgm.2009.05.2012

8. Siu CW, Yeung CY, Lau CP, Kung AWC, Tse HF: Incidence, clinical characteristics and outcome of congestive heart failure as the initial presentation in patients with primary hyperthyroidism. Heart. 2007, 93:483-487. 10.1136/hrt.2006.100628

9. Chen YT, Yang GG, Hsu YH: Thyroid storm and lymphocytic myocarditis. Intern Med. 2010, 49:593-6. 10.2169/internalmedicine.49.2504

10. Kahaly GJ, Bartalena L, Hegedüs L, Leenhardt L, Poppe K, Pearce SH: 2018 european thyroid association guideline for the management of graves' hyperthyroidism. Eur Thyroid J. 2018, 7:167-186. 10.1159/000490384

11. Umpierrez GE, Challapalli S, Patterson C: Congestive heart failure due to reversible cardiomyopathy in patients with hyperthyroidism. Am J Med Sci. 1995, 310:99-102. 10.1097/00000441-199531030-00003 